MATHEMATICS OF COMPUTATION

Volume 77, Number 264, October 2008, Pages 2437-2445

S 0025-5718(08)02093-0

Article electronically published on June 2, 2008

\title{
THE ZEROS OF DEDEKIND ZETA FUNCTIONS AND CLASS NUMBERS OF CM-FIELDS
}

\author{
GEON-NO LEE AND SOUN-HI KWON
}

\begin{abstract}
Let $F^{\prime} / F$ be a finite normal extension of number fields with Galois group $\operatorname{Gal}\left(F^{\prime} / F\right)$. Let $\chi$ be an irreducible character of $\operatorname{Gal}\left(F^{\prime} / F\right)$ of degree greater than one and $L(s, \chi)$ the associated Artin $L$-function. Assuming the truth of Artin's conjecture, we have explicitly determined a zero-free region about 1 for $L(s, \chi)$. As an application we show that, for a CM-field $K$ of degree $2 n$ with solvable normal closure over $\mathbb{Q}$, if $n \geq 370$ as well as $n \notin$ $\{384,400,416,448,512\}$, then the relative class number of $K$ is greater than one.
\end{abstract}

\section{INTRODUCTION}

For a number field $M$ we let $D_{M}, h_{M}$, and $\kappa_{M}$ be the absolute value of the discriminant, the class number of $M$, and the residue of $\zeta_{M}(s)$, the Dedekind zeta function of $M$ at $s=1$, respectively. Let $K$ be a CM-field of degree $2 n$ and let $k$ be its maximal totally real subfield. Then we have $\zeta_{K}(s)=\zeta_{k}(s) L(s, \chi)$ and $D_{K}=D_{k}^{2} f$, where $f$ is the norm of the relative discriminant. Let $h_{K}^{-}=h_{K} / h_{k}$ be the relative class number of $K$.

In [M2], Kumar Murty proved that there are only finitely many CM-fields $K$ with class number one if the normal closure $N$ of $k$ has a solvable Galois group. In this paper we determine explicitly a zero-free region about 1 for $\zeta_{K}(s)$, assuming the truth of Artin's conjecture. We apply this result to get an explicit lower bound for the relative class number of CM-fields.

Before stating our result we define the following functions as in M2. For an integer $n$, let

$$
e(n)=\max _{p^{\alpha} \| n} \alpha
$$

and

$$
\delta(n)=(e(n)+1)^{2} 3^{1 / 3} 12^{e(n)-1} .
$$

Our result is as follows.

Theorem 1. (1) Let $F^{\prime} / F$ be a normal extension of number fields with Galois group $\operatorname{Gal}\left(F^{\prime} / F\right)$. Let $\chi$ be an irreducible character of $\operatorname{Gal}\left(F^{\prime} / F\right)$ of degree larger than

Received by the editor July 6 and, in revised form, August 22, 2007.

2000 Mathematics Subject Classification. Primary 11R29, 11R42.

Key words and phrases. CM-fields, class numbers, relative class numbers, Dedekind zeta functions.

(C)2008 American Mathematical Society Reverts to public domain 28 years from publication 
one, $\mathfrak{f}_{\chi}$ its Artin conductor, and $L(s, \chi)$ the associated Artin L-function. Assume the truth of Artin's conjecture for $F^{\prime} / F$. Then $L(\sigma, \chi)$ has no real zeros in the range

$$
1-\frac{0.023932}{\chi(1)^{3} \log A_{\chi}} \leq \sigma \leq 1
$$

where $A_{\chi}=D_{F}^{\chi(1)} \mathcal{N}_{F / \mathbb{Q}}\left(\mathfrak{f}_{\chi}\right)$.

(2) Let $L / E$ be an extension of number fields of degree $n$ whose Galois closure is solvable. Suppose that $\zeta_{L}(s)$ has a real zero $\sigma$ in the range

$$
1-\frac{0.011966}{n^{e(n)} \delta(n) \log d_{L}} \leq \sigma \leq 1 .
$$

Then, there is a field $B$ with $E \subseteq B \subseteq L$ and $[B: E] \leq 2$ such that $\zeta_{B}(\sigma)=0$.

(3) Let $K$ be a $C M$-field of degree $2 n$ with solvable normal closure over $\mathbb{Q}$. Then $\zeta_{K}(\sigma)$ has no real zeros in the range

$$
1-\frac{0.000443}{n(2 n)^{e(2 n)} \delta(n)} D_{K}^{-\frac{1}{2 n}} \leq \sigma \leq 1 .
$$

In particular, if $n \geq 774$, then $\zeta_{K}(\sigma)$ has no real zeros in the range

$$
1-\frac{D_{K}^{-\frac{1}{2 n}}}{1.78244 \times 10^{40} \times(1.03412)^{n}} \leq \sigma \leq 1 .
$$

The proof is given in Section 2. We apply these estimates to get lower bounds for $h_{K}^{-}$.

Theorem 2. Let $K$ be a $C M$-field of degree $2 n$ with solvable normal closure over $\mathbb{Q}$. If $n \geq 774$, then

$$
h_{K}^{-} \geq \frac{(1.12806)^{n}}{3.04616 \times 10^{40}} \geq 1.05043>1 .
$$

If, in addition, $401 \leq n \leq 773$ and $n \notin\{405,416,432,448,480,512\}$, then we have $h_{K}^{-}>1$.

This theorem is proved in Section 3. In Section 4 we will give a better lower bound for $h_{K}^{-}$than (1.4).

\section{Proof of Theorem 1}

We use Kumar Murty's approach.

Proof of point (1). For any character $\chi$ (i.e., not necessarily irreducible) of $\operatorname{Gal}\left(F^{\prime} / F\right)$, we let $V_{\chi}$ be the underlying space of $\chi$. For each infinite place $\nu$ of $F$, denote by $\sigma_{\nu}$ the conjugacy class of Frobenius elements at primes of $F^{\prime}$ dividing $\nu$ and denote by $\chi_{\nu}^{ \pm}(1)$ the dimension of the subspace on which $\sigma_{\nu}$ acts by \pm 1 . Following Murty ([M1] and [MM]) we set

$$
a_{\chi}=\sum \chi_{\nu}^{+}(1), b_{\chi}=\sum \chi_{\nu}^{-}(1), \text { and } c_{\chi}=\chi(1) r_{2}(F),
$$

where the sums range over the real infinite places $\nu$ of $F$ and $r_{2}(F)$ is the number of complex places of $F$. Set

$$
\mathfrak{G}(s, \chi)=\left(\pi^{-s / 2} \Gamma(s / 2)\right)^{a_{\chi}}\left(\pi^{-(s+1) / 2} \Gamma((s+1) / 2)\right)^{b_{\chi}}\left((2 \pi)^{-s} \Gamma(s)\right)^{c_{\chi}}
$$

and

$$
\Lambda(s, \chi)=A_{\chi}^{s / 2} \mathfrak{G}(s, \chi) L(s, \chi)
$$


Let $\delta(\chi)=(\chi, 1)$ be the multiplicity of the trivial representation in $\chi$. We assume Artin's conjecture. Then, $(s(s-1))^{\delta(\chi)} \Lambda(s, \chi)$ is entire and, using its infinite product, we obtain that for $\operatorname{Re}(s)>1$, we have

$$
-\operatorname{Re} \frac{L^{\prime}}{L}(s, \chi) \leq-\sum_{\rho} \operatorname{Re}\left(\frac{1}{s-\rho}\right)+\frac{1}{2} \log A_{\chi}+\operatorname{Re} \frac{\mathfrak{G}^{\prime}}{\mathfrak{G}}(s, \chi)+\delta(\chi) \operatorname{Re}\left(\frac{1}{s}+\frac{1}{s-1}\right),
$$

where the sum over $\rho$ is over any subset of zeros of $\Lambda(s, \chi)$ with $0<\operatorname{Re} \rho<1$. (See [M1, p. 291] and [Od1, p. 391].) For real $\sigma$ with $1<\sigma<3 / 2, \operatorname{Re} \mathfrak{G}^{\prime} / \mathfrak{G}(\sigma, \chi)<0$ because all of the three terms $\Gamma^{\prime} / \Gamma(\sigma / 2)-\log \pi, \Gamma^{\prime} / \Gamma((\sigma+1) / 2)-\log \pi$, and $\Gamma^{\prime} / \Gamma(\sigma)-\log (2 \pi)$ are negative. Hence, for $1<\sigma<3 / 2$,

$$
-\operatorname{Re} \frac{L^{\prime}}{L}(\sigma, \chi) \leq-\sum_{\rho} \operatorname{Re}\left(\frac{1}{\sigma-\rho}\right)+\frac{1}{2} \log A_{\chi}+\delta(\chi)\left(\frac{1}{\sigma}+\frac{1}{\sigma-1}\right) .
$$

(See [M1, (2.2)].) Now we are ready to prove (1.1). From now on we assume that $\chi$ is an irreducible character of $\operatorname{Gal}\left(F^{\prime} / F\right)$ of degree larger than one. Let $\phi$ be an irreducible constituent of $\chi \otimes \bar{\chi}$ which is not the identity character or $\chi$. Let $\rho=\beta$ be a real zero of $L(s, \chi)$ with $0<\beta<1$. Following the proof of [M1, Proposition 3.7] and [HR, p. 297], we have that for $\sigma>1$,

$$
\begin{aligned}
0 \leq & -2\left(\frac{L^{\prime}}{L}(\sigma, \chi)+\frac{L^{\prime}}{L}(\sigma, \bar{\chi})\right)-\frac{\zeta_{F}^{\prime}}{\zeta_{F}}(\sigma) \\
& -\frac{L^{\prime}}{L}(\sigma, \chi \otimes \bar{\chi})-\frac{L^{\prime}}{L}(\sigma, \phi \otimes \bar{\phi})-\left(\frac{L^{\prime}}{L}(\sigma, \phi)+\frac{L^{\prime}}{L}(\sigma, \bar{\phi})\right)-\frac{I^{\prime}}{I}(\sigma),
\end{aligned}
$$

where $I(s)$ is some entire function that is real for real $s$. Since $\mathfrak{f}_{\chi \otimes \bar{\chi}}$ divides $\mathfrak{f}_{\chi}^{\bar{\chi}(1)} \mathfrak{f}_{\bar{\chi}}^{\chi(1)}=\mathfrak{f}_{\chi}^{2 \chi(1)}$ (see [Ma, p. 80] and [Od1, Lemma1]), we have $\log A_{\chi \otimes \bar{\chi}} \leq$ $2 \chi(1) \log A_{\chi}$. Similarly, $\mathfrak{f}_{\phi \otimes \bar{\phi}} \mid \mathfrak{f}_{\phi}^{2 \phi(1)}$ and $\mathfrak{f}_{\phi}\left|\mathfrak{f}_{\chi \otimes \bar{\chi}}\right| \mathfrak{f}_{\chi}^{2 \chi(1)}$, so $\log A_{\phi} \leq 2 \chi(1) \log A_{\chi}$ and $\log A_{\phi \otimes \bar{\phi}} \leq 4 \chi(1)^{3} \log A_{\chi}$.

Using (2.1), we find that for $1<\sigma<3 / 2$,

$$
\begin{aligned}
-\frac{L^{\prime}}{L}(\sigma, \chi)-\frac{L^{\prime}}{L}(\sigma, \bar{\chi}) & \leq-\frac{2}{\sigma-\beta}+\log A_{\chi}, \\
-\frac{\zeta_{F}^{\prime}}{\zeta_{F}}(\sigma) & \leq \frac{1}{\sigma}+\frac{1}{\sigma-1}+\frac{1}{2} \log A_{\chi}, \\
-\frac{L^{\prime}}{L}(\sigma, \chi \otimes \bar{\chi}) & \leq \chi(1) \log A_{\chi}+\frac{1}{\sigma}+\frac{1}{\sigma-1}, \\
-\frac{L^{\prime}}{L}(\sigma, \phi \otimes \bar{\phi}) & \leq 2 \chi(1)^{3} \log A_{\chi}+\frac{1}{\sigma}+\frac{1}{\sigma-1}, \\
-\frac{L^{\prime}}{L}(\sigma, \phi)-\frac{L^{\prime}}{L}(\sigma, \bar{\phi}) & \leq 2 \chi(1) \log A_{\chi},
\end{aligned}
$$

and $\log A_{\chi} \leq \frac{1}{2} \chi(1) \log A_{\chi} \leq \frac{1}{4} \chi(1)^{2} \log A_{\chi} \leq \frac{1}{8} \chi(1)^{3} \log A_{\chi}$. Hence,

$$
0 \leq-\frac{4}{\sigma-\beta}+\frac{3}{\sigma-1}+3 \chi(1)^{3} \log A_{\chi} .
$$

Now choosing

$$
\sigma=1+\frac{\alpha}{\chi(1)^{3} \log A_{\chi}}
$$


for a sufficiently small $\alpha>0$ gives the zero-free region

$$
1-\beta \geq \frac{-3 \alpha^{2}+\alpha}{(3 \alpha+3) \chi(1)^{3} \log A_{\chi}} .
$$

For $x>0$, the function $h(x)=\left(-3 x^{2}+x\right) /(3 x+3)$ reaches its maximum $(7-4 \sqrt{3}) / 2=0.023932 \cdots$ at $x=2 / \sqrt{3}-1=0.1547 \cdots$. Taking $\alpha=0.1547$ yields the desired zero-free region (1.1).

The point (2) follows immediately from [M2, Theorem 2.1] with $c_{1}=0.023932$.

Proof of point (3). The first statement follows from [M2, Proposition 3.2] and the proof of [M2, Theorem 3.2]. For the second statement we consider the function

$$
f(x)=2 \log _{2} x+\log _{2} x\left(\log _{2} 24+\log _{2} x\right)+2 \log _{2}\left(\log _{2} x+1\right)
$$

for $x>0$. Then

$$
f^{\prime}(x)=\frac{2+\log _{2} 24}{x \log 2}+\frac{2 \log _{2} x}{x \log 2}+\frac{2}{x \log 2\left(\log _{2} x+1\right)},
$$

which is decreasing for $x \geq e$. So, $f(x) \leq f\left(x_{0}\right)+\left(x-x_{0}\right) f^{\prime}\left(x_{0}\right)$ for $x \geq x_{0} \geq e$.

For $n \geq n_{0} \geq 3$,

$$
\begin{aligned}
n(2 n)^{e(2 n)} \delta(n) & \leq n(2 n)^{\log _{2} n+1}\left(\log _{2} n+1\right)^{2} 3^{1 / 3} 12^{\log _{2} n-1} \\
& =\left(3^{1 / 3} / 6\right) n^{2}(24 n)^{\log _{2} n}\left(\log _{2} n+1\right)^{2} \\
& =\left(3^{1 / 3} / 6\right) 2^{f(n)} \\
& \leq\left(3^{1 / 3} / 6\right) 2^{f\left(n_{0}\right)-n_{0} f^{\prime}\left(n_{0}\right)}\left(2^{f^{\prime}\left(n_{0}\right)}\right)^{n} .
\end{aligned}
$$

We evaluate $f(774)=162.0883 \cdots$ and $f^{\prime}(774)=0.048399 \cdots$, so

$$
n(2 n)^{e(2 n)} \delta(n) \leq 7.8962 \times 10^{36} \times(1.03412)^{n} \quad \text { for } n \geq 774 .
$$

Substituting (2.2) into (1.2) yields (1.3). This completes the proof of Theorem 1 . The reason why we take $n_{0}=774$ will become apparent in Section 3 below.

\section{Proof of Theorem 2}

Our proof of Theorem 2 is similar to $B$. From the analytic class number formula we have

$$
h_{K}^{-}=\frac{Q_{K} \omega_{K}}{(2 \pi)^{n}} \sqrt{\frac{D_{K}}{D_{k}}} \frac{\kappa_{K}}{\kappa_{k}} \geq \frac{2 D_{K}^{1 / 4}}{(2 \pi)^{n}} \frac{\kappa_{K}}{\kappa_{k}},
$$

where $Q_{K} \in\{1,2\}$ is the Hasse unit index of $K$ and $\omega_{K}$ denotes the number of roots of unity in $K([\mathrm{~W}])$. Using Weil's explicit formula we get a lower bound for $D_{K}$ (see (3.2) below) and an upper bound for $\kappa_{k}$ (see (3.3) below). To get a lower bound for $\kappa_{K}$ we combine Louboutin's result in [Lou and our estimate of the zero-free region about 1 for $\zeta_{K}(s)$ obtained in Section 2. Gathering together those three bounds we get an explicit lower bound for $h_{K}^{-}$. (See (3.5) below.)

For a real-valued function $F$ satisfying the conditions in [B, Proposition 3] we set

$$
I_{n}(F)=\frac{4}{n} \int_{0}^{\infty} F(x) \cosh (x / 2) d x+\int_{0}^{\infty} \frac{(1-F(x)) e^{x / 2}}{\sinh (x)} d x .
$$


We use Weil's explicit formula with

$$
F(x)= \begin{cases}9\left(\frac{\sin (x / b)-(x / b) \cos (x / b)}{(x / b)^{3}}\right)^{2} / \cosh (x / 2) & \text { for } x \neq 0 \\ 1 & \text { for } x=0\end{cases}
$$

where $b$ is the constant which is chosen so as to minimize the value $I_{n}(F)$ for a given $n$. Then we obtain a good lower bound

$$
\frac{1}{n} \log D_{k} \geq \log \left(8 \pi e^{\gamma}\right)+\frac{\pi}{2}-I_{n}(F),
$$

where $\gamma$ is an Euler constant. (For Weil's explicit formula, see [La, Ch. XVII]. For lower bounds on discriminants, see Poi1] and Poi2, Od2], and [B, Subsection 3.1].)

Remark. Integrating by parts five times and using $\int_{0}^{\infty} \frac{\sin x}{x} d x=\frac{\pi}{2}$ we obtain the following formula, which is useful to evaluate $I_{n}(F)$ :

$$
\int_{0}^{\infty}\left(\frac{\sin x-x \cos x}{x^{3}}\right)^{2} d x=\frac{\pi}{15} .
$$

In [B, Theorem 8] it was shown that for any totally real number field $k$ of degree $n \geq m$ we have

$$
\kappa_{k} \leq E_{\sigma}\left(\frac{D_{k}}{C_{5}(m)^{n}}\right)^{c_{4}(m)}
$$

where $E_{\sigma}=1$ if $\zeta_{k}(s)$ has no real zero in the range $1 / 2<\beta<1, E_{\sigma}=$ $(1-\beta) /\left(\sigma_{m}-\beta\right)$ otherwise, $\sigma_{m}$ is a constant depending only on $m$, and $c_{4}(m)$ and $C_{5}(m)$ are explicitly computable constants. The possible values for $c_{4}(m)$ and $C_{5}(m)$ for some $m$ with $400 \leq m \leq 774$ are given in Table 1. Note that $\sigma_{m}$ is not too small (i.e., $\sigma_{m}>1.001$ for $400 \leq m \leq 774$ ).

TABLE 1

\begin{tabular}{|c|c|c|c||c|c|c|c|}
\hline$m$ & $\sigma_{m}$ & $c_{4}(m)$ & $C_{5}(m)$ & $m$ & $\sigma_{m}$ & $c_{4}(m)$ & $C_{5}(m)$ \\
\hline 400 & 1.003274 & 0.339768 & 50.76955 & 448 & 1.003011 & 0.338791 & 51.37075 \\
401 & 1.003268 & 0.339746 & 50.78313 & 456 & 1.002973 & 0.338645 & 51.46186 \\
405 & 1.003244 & 0.339657 & 50.83698 & 464 & 1.002935 & 0.338504 & 51.55068 \\
408 & 1.003226 & 0.339592 & 50.87684 & 472 & 1.002898 & 0.338367 & 51.63728 \\
412 & 1.003203 & 0.339505 & 50.92936 & 480 & 1.002863 & 0.338234 & 51.72177 \\
416 & 1.003180 & 0.339421 & 50.98112 & 496 & 1.002795 & 0.337980 & 51.88471 \\
420 & 1.003158 & 0.339338 & 51.03217 & 512 & 1.002731 & 0.337739 & 52.04011 \\
424 & 1.003136 & 0.339256 & 51.08253 & 576 & 1.002507 & 0.336891 & 52.59673 \\
432 & 1.003093 & 0.339096 & 51.18119 & 640 & 1.002323 & 0.336192 & 53.06872 \\
440 & 1.003052 & 0.338941 & 51.27722 & 774 & 1.002026 & 0.335057 & 53.86114 \\
\hline
\end{tabular}

For a lower bound of $\kappa_{K}$ we use Theorem 1 in Lou (see also Theorem 16 in B]): for any totally imaginary number field $K$ of degree $\geq 10$ and root discriminant $\rho_{K} \geq 2 \pi^{2}$, we have unconditionally

$$
\kappa_{K} \geq \begin{cases}\frac{1}{c e^{1 /(2 c)} \log D_{K}} & \text { if } \zeta_{K}\left(1-1 /\left(c \log D_{K}\right)\right) \leq 0, \\ \frac{1-\beta}{2 e^{1 /(2 c)}} & \text { if } \zeta_{K}(\beta) \leq 0 \text { with } 1-1 /\left(c \log D_{K}\right) \leq \beta<1,\end{cases}
$$

where $c=(2+\sqrt{3}) / 4$. 
Set $I=\left[1-1 / c \log D_{K}, 1\right)$. We consider the three following cases:

(i) $\zeta_{k}(s)$ has a zero $\beta \in I$.

(ii) $\zeta_{k}(s)$ has no zero in $I$ and $\zeta_{K}(s)$ has a simple zero $\beta$ in $I$.

(iii) $\zeta_{k}(s)$ has no zero in $I$ and $\zeta_{K}(s)$ has no simple zero in $I$.

Case (i): If $\zeta_{k}(s)$ has a zero $\beta \in I$, then $\zeta_{K}(\beta)=0$. Note that $(1-\beta) / E_{\sigma}=$ $\sigma_{m}-\beta>\sigma_{m}-1$. From (3.1), (3.3), and (3.4), it follows that

$$
h_{K}^{-} \geq \frac{\sigma_{m}-1}{e^{1 /(2 c)}}\left(\frac{C_{5}(m)^{c_{4}(m)}}{2 \pi}\right)^{n} D_{K}^{\frac{1}{4}-\frac{1}{2} c_{4}(m)} .
$$

Case (ii): Since $\zeta_{K}(s)=\zeta_{k}(s) L(s, \chi)$, we have $L(\beta, \chi)=0$. Note that $E_{\sigma}=1$ here. Combining (3.1), (3.3), and (3.4), we have

$$
h_{K}^{-} \geq \frac{1-\beta}{e^{1 /(2 c)}}\left(\frac{C_{5}(m)^{c_{4}(m)}}{2 \pi}\right)^{n} D_{K}^{\frac{1}{4}-\frac{1}{2} c_{4}(m)} .
$$

Using the lower bound for $1-\beta$ in (1.2) we have

$$
h_{K}^{-} \geq \frac{0.000443}{e^{1 /(2 c)} n(2 n)^{e(2 n)} \delta(n)}\left(\frac{C_{5}(m)^{c_{4}(m)}}{2 \pi}\right)^{n} D_{K}^{\frac{1}{4}-\frac{1}{2} c_{4}(m)-\frac{1}{2 n}} .
$$

Case (iii): If $\zeta_{k}(s)$ has no zero in $I$ and $\zeta_{K}(s)$ has no simple zero in $I$, then either $\zeta_{K}(s)$ has no zero at all in $I$ or $\zeta_{K}(s)$ has a double zero in $I$. This is because $\zeta_{K}(s)$ has at most two zeros with multiplicity in $I$ by [LLO, Lemma 15]. Then $\zeta_{K}\left(1-1 /\left(c \log D_{K}\right)\right) \leq 0$ and

$$
h_{K}^{-} \geq \frac{2}{c e^{1 /(2 c)}}\left(\frac{C_{5}(m)^{c_{4}(m)}}{2 \pi}\right)^{n} \frac{D_{K}^{\frac{1}{4}-\frac{1}{2} c_{4}(m)}}{\log D_{K}}
$$

by (3.1), (3.3) and (3.4).

Now, we compare the three following terms:

$$
\sigma_{m}-1, \frac{0.000433 D_{K}^{-1 /(2 n)}}{n(2 n)^{e(2 n)} \delta(n)}, \frac{2}{c \log D_{K}}
$$

The second term is the smallest one among the three terms; hence 3.5 holds.

Using (3.2) with $b=6.6467$ we verify that

$$
D_{K}^{\frac{1}{2 n}} \geq D_{k}^{\frac{1}{n}} \geq 55.1658
$$

for any CM-field of degree $2 n \geq 2 \cdot 774$. Substituting (2.2), (3.6), and the values $c_{4}(774)$ and $C_{5}(774)$ in Table 1 into (3.5) yields (1.4).

In a similar way we have explicitly computed the lower bounds for $h_{K}^{-}$for all $n \leq 773$ and have verified that $h_{K}^{-}>1$ for $401 \leq n \leq 773$ except for $n=405,416$, 432, 448, 480, and 512. Our computational results are summarized in Table 2 . 
TABLE 2

\begin{tabular}{|c|c|c|c||c|c|c|c|}
\hline$n$ & $b$ & $D_{k}^{1 / n} \geq$ & $h_{K}^{-} \geq$ & $n$ & $b$ & $D_{k}^{1 / n} \geq$ & $h_{K}^{-} \geq$ \\
\hline 400 & 5.2873 & 52.2941 & 0.0000278 & 460 & 5.5516 & 52.9951 & $2.6 \times 10^{8}$ \\
401 & 5.2919 & 52.3071 & $1.4 \times 10^{11}$ & 464 & 5.5684 & 53.0368 & 1.5425 \\
402 & 5.2966 & 52.3201 & $2.1 \times 10^{8}$ & 472 & 5.6017 & 53.1184 & 109302 \\
403 & 5.3012 & 52.3330 & $2.1 \times 10^{11}$ & 480 & 5.6345 & 53.1979 & 0.001496 \\
404 & 5.3058 & 52.3459 & 14272 & 486 & 5.6589 & 53.2562 & 4.0920 \\
405 & 5.3103 & 52.3587 & 0.05238 & 488 & 5.6670 & 53.2754 & 1835091 \\
406 & 5.3149 & 52.3714 & $4.4 \times 10^{8}$ & 496 & 5.6991 & 53.3509 & 406.6 \\
407 & 5.3195 & 52.3841 & $4.2 \times 10^{11}$ & 500 & 5.7151 & 53.3880 & $1.5 \times 10^{10}$ \\
408 & 5.3241 & 52.3968 & 1.64046 & 512 & 5.7623 & 53.4964 & $6.0 \times 10^{-18}$ \\
410 & 5.3332 & 52.4220 & $8.9 \times 10^{8}$ & 520 & 5.7934 & 53.5664 & $5.4 \times 10^{8}$ \\
412 & 5.3422 & 52.4470 & 57211 & 540 & 5.8698 & 53.7345 & $2.1 \times 10^{13}$ \\
416 & 5.3603 & 52.4964 & $2.8 \times 10^{-8}$ & 544 & 5.8848 & 53.7669 & 104.2 \\
420 & 5.3782 & 52.5451 & 230434 & 576 & 6.0025 & 54.0135 & 1.6017 \\
424 & 5.3961 & 52.5930 & 25.61 & 600 & 6.0880 & 54.1847 & $1.0 \times 10^{15}$ \\
430 & 5.4226 & 52.6637 & $3.0 \times 10^{10}$ & 625 & 6.1745 & 54.3520 & $5.2 \times 10^{15}$ \\
432 & 5.4314 & 52.6869 & 0.006293 & 640 & 6.2253 & 54.4474 & 6.9917 \\
440 & 5.4663 & 52.7781 & 407.8 & 720 & 6.4833 & 54.9019 & $1.5 \times 10^{20}$ \\
448 & 5.5008 & 52.8667 & $4.3 \times 10^{-10}$ & 773 & 6.6437 & 55.1612 & $1.8 \times 10^{41}$ \\
450 & 5.5093 & 52.8885 & $4.0 \times 10^{10}$ & 774 & 6.6467 & 55.1658 & $5.5 \times 10^{36}$ \\
456 & 5.5348 & 52.9529 & 6618 & & & & \\
\hline
\end{tabular}

This completes our proof of Theorem 2 .

\section{An improvement on Theorems 1 and 2}

The second statement of Theorem 1 point (3) and Theorem 2 can be refined as follows.

Theorem 3. Let $K$ be as above.

(1) If $n \geq 726$, then $\zeta_{K}(\sigma)$ has no real zeros in the range

$$
1-\frac{D_{K}^{-\frac{1}{2 n}}}{4.06231 \times 10^{39} \times(1.0362)^{n}} \leq \sigma \leq 1 .
$$

(2) If $n \geq 726$, then

$$
h_{K}^{-} \geq \frac{(1.14136)^{n}}{3.8137 \times 10^{41}} \geq 1.2808>1 .
$$

If, in addition, $370 \leq n \leq 725$ and $n \notin\{384,400,416,448,512\}$, then we have $h_{K}^{-}>1$.

The proof of point (1) of Theorem 3 is similar to that of (1.3). To prove point (2) of Theorem 3 we proceed as in [LK]. We have used Weil's explicit formula twice: i.e., to get lower bounds for $D_{K}$ and to get upper bounds for $\kappa_{k}$. In addition we take care of prime ideals of small norms when dealing with this explicit formula of Weil. This allows us to improve especially upper bounds for $\kappa_{k}$. Analogously to the proof of Theorem 2 we can prove Theorem 3 point (2). We do not give the details of our proof, which are somewhat computational. We have explicitly computed the lower bounds for $h_{K}^{-}$for all $n \leq 725$ and give our computational results in Table 3 and Table 4. 


\section{TABLE 3}

\begin{tabular}{|c|c|}
\hline Ranges of $n$ & $n$ 's for which $h_{K}^{-}>1$ \\
\hline$n \leq 250$ & $221,223,227,229,231,233,235,237,239,241,247,249$ \\
$251 \leq n \leq 270$ & $251,253,255,257,258,259,262,263,265,266,267,269$ \\
$271 \leq n \leq 285$ & $271,273,274,277,278,279,281,282,283,285$ \\
$286 \leq n \leq 300$ & $286,287,289,290,291,293,294,295,298,299$ \\
$301 \leq n \leq 330$ & all $n$ 's except for 304,308,312,320,324,328 \\
$331 \leq n \leq 370$ & all $n$ 's except for 336,344,352,360,368 \\
$371 \leq n \leq 725$ & all $n$ 's except for 384,400,416,448, 512 \\
\hline
\end{tabular}

TABLE 4

\begin{tabular}{|c|c||c|c||c|c|}
\hline$n$ & $h_{K}^{-} \geq$ & $n$ & $h_{K}^{-} \geq$ & $n$ & $h_{K}^{-} \geq$ \\
\hline 370 & $3.7 \times 10^{8}$ & 400 & 0.0160 & 480 & 1.3725 \\
371 & $3.2 \times 10^{11}$ & 410 & $5.3 \times 10^{11}$ & 500 & $1.5 \times 10^{13}$ \\
372 & 27570 & 416 & $1.7 \times 10^{-5}$ & 512 & $6.4 \times 10^{-15}$ \\
373 & $4.8 \times 10^{11}$ & 420 & $1.4 \times 10^{8}$ & 520 & $6.1 \times 10^{11}$ \\
374 & $7.7 \times 10^{8}$ & 430 & $2.1 \times 10^{13}$ & 540 & $2.6 \times 10^{16}$ \\
375 & 2202 & 440 & 296533 & 600 & $1.6 \times 10^{18}$ \\
380 & 114506 & 448 & $3.2 \times 10^{-7}$ & 625 & $9.3 \times 10^{18}$ \\
384 & $4.8 \times 10^{-16}$ & 450 & $2.9 \times 10^{13}$ & 640 & 13578 \\
390 & $1.4 \times 10^{10}$ & 460 & $2.1 \times 10^{11}$ & 720 & $4.0 \times 10^{23}$ \\
\hline
\end{tabular}

\section{ACKNOWLEDGMENTS}

G.-N. Lee was supported by a Korea University Grant and S.-H. Kwon was supported by KRF-2005-C00002.

\section{REFERENCES}

[B] S. Bessassi, Bounds for the degrees of CM-fields of class number one, Acta Arith., 106, 3(2003), 213-245. MR1957106 (2003m:11183)

[HR] J. Hoffstein and D. Ramakrishnan, Siegel zeros and cusp forms, Intl. Math. Res. Not. 6 (1995), 279-308. MR1344349(96h:11040)

[La] S. Lang, Algebraic number theory, Second Edition, Springer-Verlag, 1994. MR 1282723 (95f:11085)

[LK] G.-N. Lee and S.-H. Kwon, CM-Fields with relative class number one, Math. Comp., 75 (2006), 997-1013. MR.2197004 (2006k:11216)

[LLO] F. Lemmermeyer, S. Louboutin, and R. Okazaki, The class number one problem for some non-abelian normal CM-fields of degree 24, J. Théor. Nombres Bordeaux, 11(1999), 387406. MR 1745886 (2001j:11104)

[Lou] S. Louboutin, Explicit lower bounds for residues at $s=1$ of Dedekind zeta functions and relative class numbers of CM-fields, Trans. Amer. Math. Soc., 355(2003), 3079-3098. MR.1974676(2004f:11134)

[M1] V. Kumar Murty, Modular forms and the Chebotarev density theorem, II, Analytic Number Theory (Kyoto, 1996), 287-308, London Math. Soc. Lecture Note Ser., 247, Cambridge Univ. Press, Cambridge, 1997. MR1694997 (2000g:11094)

[M2] V. Kumar Murty, Class numbers of CM-fields with solvable normal closure, Composito Mathematica 127 (2001), 273-287. MR1845038 (2003a:11147)

[Ma] J. Martinet, Character theory and Artin L-functions, in Algebraic Number Fields (Lfunctions and Galois properties), edited by A. Fröhlich, Academic Press, 1977, pp 1-87. MR0447187(56:5502) 
[MM] M. Ram Murty and V. Kumar Murty, Non-vanishing of L-functions and applications, Progress in Mathematics, vol. 157, Birkhäuser, 1997. MR 1482805 (98h:11106)

[Od1] A. M. Odlyzko, On Conductors and Discriminants, in Algebraic Number Fields (Lfunctions and Galois properties), edited by A. Fröhlich, Academic Press, 1977, pp. 377-407. MR 0453701 (56:11961)

[Od2] A. M. Odlyzko, Bounds for discriminants and related estimates for class numbers, regulators and zeros of zeta functions: A survey of recent results, Sém. Théor. Nombres Bordeaux 2 (1990), 119-141. MR.1061762 (91i:11154)

[Poi1] G. Poitou, Minorations de discriminants, Sém. Bourbaki, 28e année (1975/76), 136-153. MR0435033 (55:7995)

[Poi2] G. Poitou, Sur les petits discriminants, Sém. Delange-Pisot-Poitou, 18e année, 1976/77. MR551335 (81i:12007)

[W] L. C. Washington, Introduction to cyclotomic fields, 2nd ed. (1997), Grad. Texts in Math. 83, Springer-Verlag. MR.1421575 (97h:11130)

Department of Mathematics, Korea University, 136-701, Seoul, Korea

E-mail address: thisknow@korea.ac.kr

Department of Mathematics Education, Korea University, 136-701, Seoul, Korea

E-mail address: sounhikwon@korea.ac.kr 